\title{
Robust Asynchronous STBC Transceiver for Multiple-Access Frequency-Selective Channels
}

\author{
Dandan Wang, Hlaing Minn and Naofal Al-Dhahir \\ Dept of Electrical Engineering, University of Texas at Dallas, Richardson, TX 75083 \\ Email: dxw053000,hlaing.minn,aldhahir@utdallas.edu
}

\begin{abstract}
1 Abstract-In this paper, we propose a robust STBC transmission scheme to combat the timing synchronization errors over frequency-selective multiple-access channels. First, the equivalent channel model in the presence of timing synchronization errors is derived and we find that the synchronization errors result in an equivalent channel model with larger number of correlated channel taps. Based on this correlated equivalent channel model, the statistical power profile is analyzed and a robust statistical bit loading algorithm is proposed. At the receiver, by exploiting the algebraic structure of the Alamouti STBC, a closed-form expression of the $Q R$ receiver is derived which further simplifies computational complexity. Simulation results show that our proposed scheme is robust to timing synchronization errors.
\end{abstract}

\section{INTRODUCTION}

Multiple input multiple output (MIMO) systems take advantage of the spatial diversity available using spatially separated antennas in a multipath scattering environment. Since wireless communication is challenged by limited spectral resources, frequency reuse and multi-user multiplexing have received considerable attention. Recently, [1] demonstrated that multi-user MIMO systems can have better performance in terms of maximum throughput than time-division-multiple-access-based schemes via transmit signal processing if the number of transmit antennas is much larger than the number of receive antennas. In this context, a space-time transceiver has been investigated in [6] over flat-fading channels assuming two users perfectly time synchronized. However, in practical systems, it is very difficult, if not impossible, to get perfect synchronization among all uplink users. In [5], the authors consider an equivalent channel model for a flat fading system with synchronization errors in a cooperative network. Since in broadband systems, the channels are usually frequency-selective, this motivates us to investigate the multi-user MIMO transceiver with synchronization errors over frequency-selective channels.

In this paper, we first derive the equivalent channel model in the presence of synchronization errors and find that the effect of synchronization errors is to extend the

\footnotetext{
${ }^{1}$ This work was supported in parts by the Erik Jonsson School Research Excellence Initiative, the University of Texas at Dallas, USA the Texas Advanced Technology Program (ATP) under Contract 0097410023-2003, the National Science Foundation (NSF) under Contracts CCF 04-30654 and DMS 05-28010, and a gift from Texas Instruments Inc.
}

length of the original channel impulse response (CIR) and introduce correlation among its taps. For frequencyselective channels, space-time block codes (STBC) have been combined with orthogonal frequency division multiplexing (OFDM) by implementing STBC at a block level instead of symbol level, namely ST-OFDM [2]. Based on the special structure of the statistical channel profile introduced by the equivalent channel model, we propose a robust transmission scheme to combat the synchronization errors. At the receiver, by exploiting the speical algebraic structure of STBC in [7], we propose a closed-form onestep QR space-time detector which outperforms the 2-step MMSE interference cancellation algorithm in [6].

The paper is organized as follows. In Section II, the system set-up and the equivalent channel model with synchronization errors are presented. The statistical power profile of the equivalent channel model and a robust statistical bit loading algorithm are presented in Section III. A closed-form QR detector is described in Section IV. In Section V, simulation results are presented and conclusions are drawn in Section VI.

\section{SySTEM MODEL}

\section{A. System Set-up}

In this paper, we consider an uplink system, in which multiple users access the base station at the same time within the same bandwidth using spatial division multiple access (SDMA). Each user has two transmit antennas and there are multiple receive antennas at the base station (to decode the information from different users, the number of receive antennas should be at least equal to the number of users). We use the Alamouti STBC in a ST-OFDM system with $N$ sub-carriers. At the base station, FFT is first implemented and then an interference cancellation scheme (which is called STBC-IC detector) is adopted on each sub-carrier to decode the independent information streams from different users. We consider a system with timing synchronization errors, i.e. signals from different users arrive at the base station with different delays. Fig. 1 illustrates the scenario of two users access to the base station with two receive antennas.

\section{B. Equivalent Channel Model}

In this subsection, we will derive the equivalent multipath channel model with synchronization errors. The 


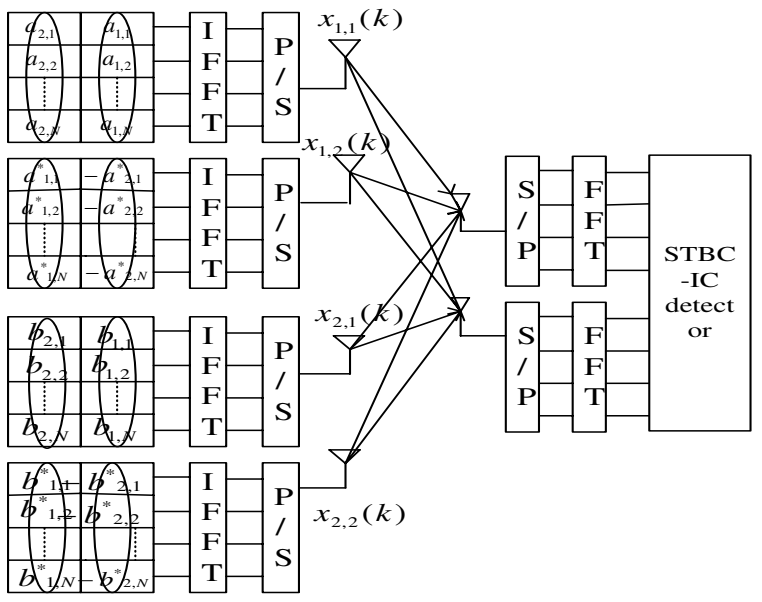

Fig. 1. System set-up

distribution of the channel taps between different transmit and receiver antenna pairs are assumed to be the same. The transmitted signals from the $j$ th transmit antenna of the $i$ th user in the time domain are $x_{i, j}(k)$ with $i, j=1,2$ and $k=1,2, \ldots$. The transmitted signals $d_{i, j}(t), i, j=$ 1,2 are

$$
d_{i, j}(t)=\sum_{k=-\infty}^{\infty} x_{i, j}(k) \delta(t-k T) ; i, j=1,2,
$$

where $T$ is the symbol period ${ }^{2}$. The channel between the $j$ th antenna of the $i$ th user and the first receive antenna is

$$
g_{i, j}(t)=\sum_{l=1}^{L_{i, j}} g_{i, j, l} \delta\left(t-\tau_{i, j, l} T\right),
$$

where $g_{i, j, l}$ are the channel tap gains with different delays. Denote the combined transmit and receive pulse shaping filter as $p(t)$. Then, at the first receive antenna, the signal received from the $j$ th antenna of the $i$-th user is

$$
\begin{aligned}
r_{i, j}(t) & =d_{i, j}(t) \otimes g_{i, j}(t) \otimes p(t) \\
& =\sum_{k=-\infty}^{\infty} x_{i, j}(k) \sum_{l=1}^{L_{i, j}} g_{i, j, l} p\left(t-\tau_{i, j, l} T-k T\right) .
\end{aligned}
$$

Let $t_{i, j}$ denote the timing error with respect to the ideal samples for the $n_{i, j}$ th symbol of the $j$-th antenna of the $i$-th user. Then, the sampling time for the $n_{i, j}$ th symbol of user $i$ on the $j$ th antenna is $n_{i, j} T+t_{i, j}$, and the corresponding sample is

$$
\begin{aligned}
& r_{i, j}\left(n_{i, j} T+t_{i, j}\right) \\
& =\sum_{k=-\infty}^{\infty} x_{i, j}(k) \sum_{l=1}^{L_{i, j}} g_{i, j, l} p\left(n_{i, j} T+t_{i, j}-\tau_{i, j, l} T-k T\right) \\
& =\sum_{l=1}^{L_{i, j}} g_{i, j, l} \sum_{k=-\infty}^{\infty} x_{i, j}(k) p\left(\left(n_{i, j}-\tau_{i, j, l}\right) T+t_{i, j}-k T\right)
\end{aligned}
$$

Let $p(t)$ be the raised cosine pulse shape function given as

$$
p(t)=\frac{\sin (\pi t / T)}{\pi t / T} \frac{\cos (\alpha \pi t / T)}{1-4 \alpha^{2} t^{2} / T^{2}}
$$

\footnotetext{
${ }^{2} \frac{1}{T}$ is $N$ times the sub-carrier spacing
}

and we only consider the first-order side lobes. If $0<$ $t_{i, j}<T$, (4) is changed into

$$
\begin{aligned}
& r_{i, j}\left(n_{i, j} T+t_{i, j}\right)=\sum_{l=1}^{L_{i, j}}\left[x_{i, j}\left(n_{i, j}-\tau_{i, j, l}\right) g_{i, j, l} p\left(t_{i, j}\right)\right. \\
& +x_{i, j}\left(n_{i, j}-\tau_{i, j, l}-1\right) g_{i, j, l} p\left(t_{i, j}+T\right) \\
& \left.+x_{i, j}\left(n_{i, j}-\tau_{i, j, l}+1\right) g_{i, j, l} p\left(t_{i, j}-T\right)\right] .
\end{aligned}
$$

When there is no timing error, i.e. $t_{i, j}=0$,

$$
r_{i, j}\left(n_{i, j} T\right)=\sum_{l=1}^{L_{i, j}} x_{i, j}\left(n_{i, j}-\tau_{i, j, l}\right) g_{i, j, l} .
$$

Comparing (6) and (7), we can see that the timing errors extend the length of the original channel taps and the equivalent extended channel taps are correlated no matter the original channel taps are independent or not. To further get the essence of the effects of the timing errors, we consider an originally independent sample-spaced channel taps.

1) Example: For $i=1, j=1$, we assume $L_{1,1}=3$, $\tau_{1,1,1}=0, \tau_{1,1,2}=1, \tau_{1,1,3}=2$, then

$$
\begin{aligned}
& r_{1,1}\left(n_{1,1} T+t_{1,1}\right) \\
& =g_{1,1,1}\left[x_{1,1}\left(n_{1,1}\right) p\left(t_{1,1}\right)+x_{1,1}\left(n_{1,1}-1\right) p\left(t_{1,1}+T\right)\right. \\
& \left.+x_{1,1}\left(n_{1,1}+1\right) p\left(t_{1,1}-T\right)\right]+g_{1,1,2}\left[x_{1,1}\left(n_{1,1}-1\right) p\left(t_{1,1}\right)\right. \\
& \left.+x_{1,1}\left(n_{1,1}-2\right) p\left(T+t_{1,1}\right)+x_{1,1}\left(n_{1,1}\right) p\left(t_{1,1}-T\right)\right] \\
& +g_{1,1,3}\left[x_{1,1}\left(n_{1,1}-2\right) p\left(t_{1,1}\right)+x_{1,1}\left(n_{1,1}-3\right) p\left(t_{1,1}+T\right)\right. \\
& \left.+x_{1,1}\left(n_{1,1}-1\right) p\left(t_{1,1}-T\right)\right] .
\end{aligned}
$$

Then, the equivalent channel vector is $\mathbf{h}_{1,1}=$ $\left[g_{1,1,1} p\left(t_{1,1}-T\right), g_{1,1,1} p\left(t_{1,1}\right)+g_{1,1,2} p\left(t_{1,1}-\right.\right.$ $T), g_{1,1,1} p\left(t_{1,1}+T\right)+g_{1,1,2} p\left(t_{1,1}\right)+g_{1,1,3} p\left(t_{1,1}-\right.$ $\left.T), g_{1,1,2} p\left(t_{1,1}+T\right)+g_{1,1,3} p\left(t_{1,1}\right), g_{1,1,3} p\left(t_{1,1}+T\right)\right]^{T}$. In this paper, $(.)^{T}$ means transpose and $(.)^{H}$ means transpose conjugate. Let $p_{0}=p\left(t_{1,1}-T\right), p_{1}=p\left(t_{1,1}\right)$, and $p_{2}=p\left(t_{1,1}+T\right)$ and define

$$
\mathbb{P}_{1}=\left(\begin{array}{ccc}
p_{0} & 0 & 0 \\
p_{1} & p_{0} & 0 \\
p_{2} & p_{1} & p_{0} \\
0 & p_{2} & p_{1} \\
0 & 0 & p_{2}
\end{array}\right) \text { and } \mathbf{g}_{1,1}=\left(\begin{array}{c}
g_{1,1,1} \\
g_{1,1,2} \\
g_{1,1,3}
\end{array}\right) \text {. }
$$

Then, $\mathbf{h}_{1, j}=\mathbb{P}_{1} * \mathbf{g}_{1,1}$.

\section{ROBUST STATISTICAL BIT LOADING SCHEME}

From the previous section, we observe that the synchronization errors render the original channel taps into longer correlated channel taps. We show next how to exploit knowledge of this correlation profile to combat the effect of synchronization errors through statistical bit loading.

\section{A. Statistical power profile}

In the following, the statistical power profile of this equivalent channel model is analyzed. Let $\mathbf{H}_{i, j}=$ $\left[H_{i, j, 1}, H_{i, j, 2}, \ldots, H_{i, j, N}\right]^{T}, i, j=1,2$ denote the frequency domain channel response of the equivalent correlated channel tap vector $\mathbf{h}_{i, j}$. Denote $M_{i, j}$ as the length of $\mathbf{h}_{i, j}$. Without loss of generality, we consider the channel between the first antenna of user 1 and the first receiver 
antenna. The covariance matrix of the channel frequency responses is

$$
\mathbb{C}=E\left(\mathbf{H}_{1,1} \mathbf{H}_{1,1}^{H}\right)=E\left(\mathbb{V} \mathbf{h}_{1,1} \mathbf{h}_{1,1}^{H} \mathbb{V}^{H}\right)=\mathbb{V} \mathbb{R} \mathbb{V}^{H}
$$

where $\mathbb{R}=E\left(\mathbf{h}_{1,1} \mathbf{h}_{1,1}^{H}\right)$ and $\mathbb{V}$ is the truncated DFT matrix whose $(n, m)$ element is defined as

$$
V_{n, m}=e^{-\frac{j 2 \pi(n-1)(m-1)}{N}} ; n=1,2, \ldots N, m=1,2, \ldots, M_{i, j}
$$

Now, let us check the average power on each subcarrier, i.e. the diagonal elements of $\mathbb{C}$. From (10), we obtain that

$$
\begin{aligned}
C_{n, n} & =\sum_{k=1}^{M_{1,1}} \sum_{m=1}^{M_{1,1}} R_{m, k} V_{n, m} V_{n, k}^{*} \\
& =\sum_{m=1}^{M_{1,1}} R_{m, m}+\sum_{k-m \neq 0} R_{m, k} e^{\frac{j 2 \pi(n-1)(k-m)}{N}},
\end{aligned}
$$

where $R_{m, k}$ is the $(m, k)$ element of $\mathbb{R}$.

From (12), we can see that if $\mathbb{R}$ were a diagonal matrix, i.e. $\mathbf{h}_{1,1}$ had independent channel taps, we would have $C_{n, n}=\sum_{m=1}^{M_{1,1}} R_{m, m}$ indicating the same average power on all sub-carriers. However, the correlation among the equivalent channel taps makes the average power on different sub-carriers unequal. We can see this more clearly from the example used in the previous section.

1) Example in Section II. B: Assume that the original channel taps $g_{1, j, 1}, g_{1, j, 2}$ and $g_{1, j, 3}$ are independent, i.e.

$$
R_{\mathrm{g}_{1,1}}=\left(\begin{array}{ccc}
\sigma_{1}^{2} & 0 & 0 \\
0 & \sigma_{2}^{2} & 0 \\
0 & 0 & \sigma_{3}^{2}
\end{array}\right) .
$$

Then,

$$
\mathbb{R}=\mathbb{E}\left(\mathbf{h}_{1,1} \mathbf{h}_{1,1}^{H}\right)=E\left(\mathbb{P}_{1} \mathbf{g}_{1,1} \mathbf{g}_{1,1}^{H} \mathbb{P}_{1}^{H}\right)=\mathbb{P}_{1} \mathbb{R}_{g_{1,1}} \mathbb{P}_{1}^{H}
$$

Substituting (9), (13) and (14) into (12), we obtain

$$
\begin{aligned}
C_{n, n} & =\left(\sum_{l=1}^{3} \sigma_{l}^{2}\right)\left\{2\left(p_{0} p_{1}+p_{1} p_{2}\right) \cos \left(\frac{2 \pi(n-1)}{N}\right)\right. \\
& \left.+2 p_{0} p_{2} \cos \left(\frac{2 \pi(n-1) * 2}{N}\right)+p_{0}^{2}+p_{1}^{2}+p_{2}^{2}\right\} .
\end{aligned}
$$

The early-late gate is a simple and practical method that is used widely for symbol synchronization and sampling at the receiver [8]. Let $\tau_{1}$ and $\tau_{2}$ denote the delays from user 1 and user 2 respectively. If $\tau=\tau_{1}-\tau_{2}$ is a multiple of the symbol interval $T$, which is the symbol interval after parrel to serial transformation, the sampling time decided by the gate is ideal for both signals. Without loss of generality, we now assume $0 \leq|\tau| \leq T$. In [5], it is shown that the sampling time decided by the gate is about $\frac{\tau}{2}$ ahead of the ideal time for one signal and $\frac{\tau}{2}$ behind the ideal time for the other, i.e $t_{1,1}=\frac{\tau}{2}$. The power profiles with different synchronization errors $\tau$ obtained from (15) are shown in Fig. 2. In practical systems, $\tau$ is unknown at the receiver. Thus, we can only average the power profile over all possible $\tau$. In this paper, we assume that $\tau$ is a random variable with probability density function $f(\tau)$, then the average power profile is obtained by averaging (15) over $\tau$ as

$$
\bar{C}_{n, n}=\int_{-\infty}^{\infty} C_{n, n} f(\tau) d \tau
$$

where $C_{n, n}$ is implicitly a function of $\tau$. The average power profile with $\tau$ uniformly distributed over $[0, T]$ and $N=64$ is shown in Fig. 2 .

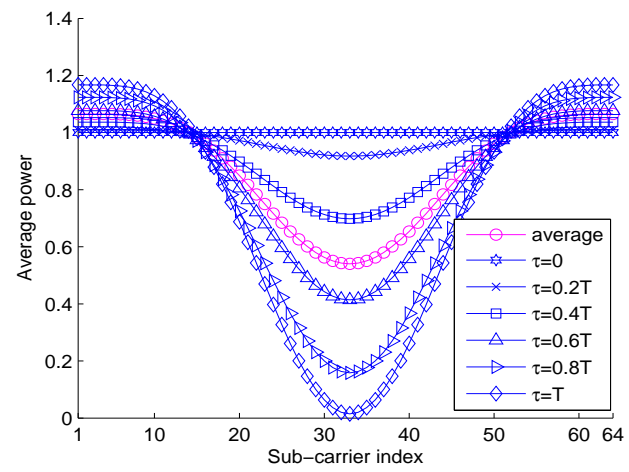

Fig. 2. Average power on different sub-carriers for different synchronization errors

\section{B. Statistical bit loading scheme}

In the previous subsection, the statistical channel power profile is derived. In this subsection, we will present a statistical bit loading scheme to fully utilize this statistical channel power profile. A bit loading scheme, typically used in wireline systems, assigns a high data rate to sub-carriers with high SNR and a low data rate to subcarriers with low SNR. In wireless systems, it is difficult to obtain the instantaneous channel information for the fast time-varying channels at the transmitter and thus it is not not feasible to adopt an adaptive bit loading algorithm. However, as shown in Fig. 2, the average power profile has very special structure. Although we do not know the instantaneous channel information, it can be seen that there are some sub-carriers which have less average power than the other sub-carriers. With this average power profile, we propose a statistical bit loading scheme. The major difference between our proposed statistical bit loading scheme and the bit loading scheme for discrete multitone transmission presented it [4] is that the normalized noise power in [4] is replaced by the ratio of the noise power to the average channel power gain on different sub-carriers numerically evaluated in (16). Our objective is to minimize the average bit error rate (BER) over all sub-carriers with the total bit rate held fixed. In the following, we summarize our proposed statistical bit loading scheme in five steps:

1) Step 1: Select active sub-carriers

$$
R_{i}=\frac{R_{T}}{|I|}+\frac{1}{|I|} \log _{2}\left(\frac{\prod_{l \in I} W_{l}}{W_{i}^{|I|}}\right)
$$

where $R_{T}$ is the target bit rate over all sub-carriers and $W_{i}=\frac{N_{i}}{P_{i}}$ with $N_{i}$ being the noise power on the $i$ th sub-carrier and $P_{i}$ being the average channel power gain on the $i$ th sub-carrier. Initially, $I=\{1,2, \ldots, N\} .|I|$ denotes the size of the set $I$. If $R_{i}<0$, then remove $i$ from $I$. This is done iteratively until all rates of the remaining subcarriers are positive. 
2) Step 2: $R_{i}$ is quantized to $R_{Q, i}$ with

$$
R_{Q i}= \begin{cases}R_{\max } & R_{i}>=R_{\max }-0.5 \\ \text { Floor }\left(R_{i}+0.5\right) & 0.5<R_{i}<R_{\max }-0.5 \\ 0 & R_{i}<0.5,\end{cases}
$$

where $R_{\max }$ is the predefined maximum constellation size on a sub-carrier.

3) Step 3: If $\sum_{i \in I} R_{Q i},<(>) R_{T}$, the rate of the channel with the largest (smallest) $\Delta R_{i}=R_{i}-$ $R_{Q i}$, is incremented (decremented). The initial bit allocation stops if $\sum_{i \in I} R_{Q i}=R_{T}$.

4) Step 4: Based on the results from Step 3, adjust the bit allocation on the sub-carriers with $R_{Q i}=1 \mathrm{such}$ that half of these sub-carriers with higher (lower) power profile are assigned with $R_{Q i}=2\left(R_{Q i}=0\right)$ so that $\sum_{i \in I} R_{Q i}=R_{T}$. If the number of subcarriers with $R_{Q i}=1$ is odd, then reduce the modulation size of the sub-carriers with smallest average power among those with the highest modulation scheme by '1' bit.

5) Step 5: Transmit power is assigned to the subcarriers recorded in the set $I$ so that all sub-carriers yield the same error probability, i.e.

$$
S_{i}=\frac{S_{T} W_{i} 2^{R_{Q i}}}{\sum_{l \in I} W_{l} 2^{R_{Q l}}}
$$

where $S_{T}$ is the fixed total transmission power.

We have the following two remarks about the results:

- For Alamouti space-time coding, two antennas from the same user are designed to have the same bit loading scheme. It would be interesting to investigate other space-time codes which can have different modulation schemes on different antennas.

- So far, we have assumed that the timing error is $0<t_{i, j}<T$. When the timing error is $D=$ $d T+t_{i, j}$, where $d$ is an integer and $0<t_{i, j}<T$, the shift in time domain corresponds to a phase change in frequency domain. We need to add $d$ more cyclic prefix symbols to avoid the interference among different OFDM blocks.

\section{INTERFERENCE CANCELLATION SCHEME}

In the previous sections, we showed that the effects of synchronization errors increase the length and introduce correlation between CIR taps. Therefore, at the transmitter, the length of the cyclic prefix $(\mathrm{CP})$ should also be extended so that the interference among different OFDM symbols can be avoided. At the transmitter, we first implement the statistical bit loading scheme and then implement ST-OFDM. The receiver is assumed to know the result of the statistical bit loading scheme. The channels are assumed to be constant over two continuous OFDM blocks. We consider that two users access to the base station and there are two receive antennas at the base station. Then the received signals on the $n$th sub-carrier can be written as

$$
\mathbf{y}=\mathbb{H} * \mathbf{x}+\mathbf{Z}
$$

where $\mathbf{y}=\left[y_{1,1, n},-y_{1,2, n}^{*}, y_{2,1, n},-y_{2,2, n}^{*}\right]^{T}$ is the received signal vector and $\mathbf{x}=\left[a_{1, n}, a_{2, n}, b_{1, n}, b_{2, n}\right]^{T}$ is the transmitted signal vector of these two users. $\mathbf{Z}=$ $\left[N_{1,1, n}, N_{1,2, n}, N_{2,1, n}, N_{2,2, n}\right]^{T}$ is the noise vector on $n$th sub-carrier. $\mathbb{H}$ is the space-time equivalent channel model on the $n$th sub-carrier given as

$$
\mathbb{H}=\sqrt{S_{n}} *\left(\begin{array}{cccc}
H_{1,1, n} & G_{1,1, n} & H_{2,1, n} & G_{2,1, n} \\
-G_{1,1, n}^{*} & H_{1,1, n}^{*} & -G_{2,1, i}^{*} & H_{2,1, n}^{*} \\
H_{1,2, n} & G_{1,2, n} & H_{2,2, n} & G_{2,2, n} \\
-G_{1,2, n}^{*} & H_{1,2, n}^{*} & -G_{2,2, n}^{*} & H_{2,2, n}^{*}
\end{array}\right),
$$

where, $H_{i, j, n}, i, j=1,2, n=1,2, \ldots, N$ is the channel response on the $n$th sub-carrier from the $j$ th antenna of user $i$ to the first receive antenna while $G_{i, j, n}$ is to the second receive antenna correspondingly. As $\mathbb{H}$ consists of four Alamouti matrix [9], we obtain a closed-form QR decomposition of $\mathbb{H}$ as in the following theorem:

Theorem: For a matrix $\mathbb{H}=\left(\begin{array}{cc}A & B \\ C & D\end{array}\right)$, in which $\mathrm{A}, \mathrm{B}, \mathrm{C}$ and $\mathrm{D}$ are all Alamouti matrixes, i.e. $A=\left(\begin{array}{cc}a_{1} & a_{2} \\ -a_{2}^{*} & a_{1}^{*}\end{array}\right), B=\left(\begin{array}{cc}b_{1} & b_{2} \\ -b_{2}^{*} & b_{1}^{*}\end{array}\right), C=$ $\left(\begin{array}{cc}c_{1} & c_{2} \\ -c_{2}^{*} & c_{1}^{*}\end{array}\right)$ and $D=\left(\begin{array}{cc}d_{1} & d_{2} \\ -d_{2}^{*} & d_{1}^{*}\end{array}\right)$, the QR decomposition of $\mathbb{H}$ is

$$
Q_{H}=\frac{1}{\sqrt{\alpha_{A}+\alpha_{C}}}\left(\begin{array}{cc}
A & -\sqrt{\frac{\alpha_{C}}{\alpha_{A}}} \frac{1}{\sqrt{\alpha_{F}}} A F^{H} \\
C & \sqrt{\frac{\alpha_{A}}{\alpha_{C}}} \frac{1}{\sqrt{\alpha_{F}}} C F^{H}
\end{array}\right)
$$

and

$$
R_{H}=\left(\begin{array}{cc}
\sqrt{\alpha_{A}+\alpha_{C}} I_{2} & \frac{A^{H} B+C^{H} D}{\sqrt{\alpha_{A}+\alpha_{C}}} \\
0 & \sqrt{\alpha_{F}} I_{2}
\end{array}\right),
$$

where $F=\frac{\sqrt{\frac{\alpha_{A}}{\alpha_{C}}} D^{H} C-\sqrt{\frac{\alpha_{C}}{\alpha_{A}}} B^{H} A}{\sqrt{\alpha_{A}+\alpha_{C}}}=\left(\begin{array}{cc}f_{1} & f_{2} \\ -f_{2}^{*} & f_{1}^{*}\end{array}\right)$, $\alpha_{F}=\left|f_{1}\right|^{2}+\left|f_{2}\right|^{2}, \quad \alpha_{A}=\left|a_{1}\right|^{2}+\left|a_{2}\right|^{2}, \quad$ and $\alpha_{C}=\left|c_{1}\right|^{2}+\left|c_{2}\right|^{2}$.

This closed-form $\mathrm{QR}$ expression utilizes the special structure of Alamouti space-time codes and it saves almost half of the computational complexity compared with the general approaches of QR decomposition, such as Givens rotation. After we obtain the QR decomposition of $\mathbb{H}$, multiplying $Q^{H}$ to the both sides of (20), we obtain

$Q^{H} y=R_{H} \mathbf{x}+\mathbf{Z}^{\prime}$

$=\left(\begin{array}{cccc}\sqrt{\alpha_{A}+\alpha_{C}} & 0 & e_{1}^{*} & -e_{2} \\ 0 & \sqrt{\alpha_{A}+\alpha_{C}} & e_{2}^{*} & e_{1} \\ 0 & 0 & \sqrt{\alpha_{F}} & 0 \\ 0 & 0 & 0 & \sqrt{\alpha_{F}}\end{array}\right)\left(\begin{array}{c}a_{1, n} \\ a_{2, n} \\ b_{1, n} \\ b_{2, n}\end{array}\right)+\mathbf{Z}^{\prime}$,

where $\mathbf{Z}^{\prime}=Q^{H}\left[N_{1,1, n}, N_{1,2, n}, N_{2,1, n}, N_{2,2, n}\right]^{T}$.

From (24), we can first decode $b_{1, n}$ and $b_{2, n}$, then remove the interference of $b_{1, n}$ and $b_{2, n}$ to $a_{1, n}$ and $b_{2, n}$, which is the iterative procedure of $\mathrm{QR}$ detector. It is noteworthy that our closed-form QR decomposition can be used not only for QR detector, but also for any other detector involving the calculation of $\mathrm{QR}$ decomposition.

\section{SIMULATION RESULTS}

In our simulations, the channels are modelled as independent 3-tap Rayleigh fading channels with an exponential power delay profile. The equivalent channels impaired by the timing synchronization errors are assumed to be perfectly known at the receiver. The synchronization 
errors are assumed to be uniformly distributed over $(0, T)$. We consider ST-OFDM systems with $N=64$ subcarriers. The detection of ST-OFDM is done per subcarrier, i,e, STBC-IC detector is implemented on each sub-carrier. We compare the following four STBC-IC detection schemes:

- Linear MMSE: the scheme proposed in [6] is implemented on each sub-carrier.

- QR: the transmitted signal on each sub-carrier is detected iteratively using the closed-form QR decomposition of $\mathbb{H}$.

- Ordered QR: According to the effective channel power gains of different signals (i.e, the summation of the absolute value of different columns of $\mathbb{H}$ ), reorder $\mathbb{H}$ so that the signal with the largest effective channel power gain is decoded first.

- Lattice-aided reduction receiver (LR): In [3], a lattice-aided reduction receiver is presented for a VBLAST system. In fact, the received signal model in (20) can be viewed as an equivalent 4 by 4 V-BLAST system with a special channel model. Thus, the LR receiver in [3] can be used as the STBC-IC detector. Also, since the first step of LR is to perform QR decomposition of $\mathbb{H}$, our proposed closed-form QR can be used to simplify the computation complexity of LR.

In Fig. 3, we compare the performance of these four STBC-IC detection schemes with the statistical bit loading scheme. It can be seen that the QR detector has almost the same performance as the linear MMSE detector. However, with ordering, about $1 \mathrm{~dB}$ gain can be obtained at $\mathrm{BER}=10^{-4}$ while LR can achieve about $3 \mathrm{~dB}$ gain. Also, the slope of LR is different from the other schemes, which shows that LR can achieve a higher diversity order. In Fig. 4, the advantage of our proposed statistical bit loading algorithm is shown with LR detector, where it can be seen that the statistical bit loading algorithm has about $3.5 \mathrm{~dB}$ gain at $\mathrm{BER}=10^{-4}$. Fig. 4 also gives the performance without synchronization errors, which means the average power on all the sub-carriers are the same and no statistical bit loading scheme is needed. Thus, we can see that the statistical bit loading scheme is more robust to the timing synchronization errors compared with the conventional scheme without statistical bit loading.

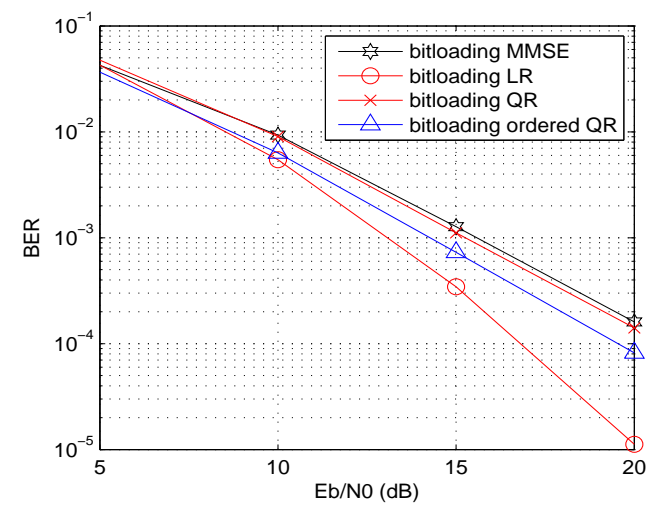

Fig. 3. Performance comparison with different STBC-IC detectors

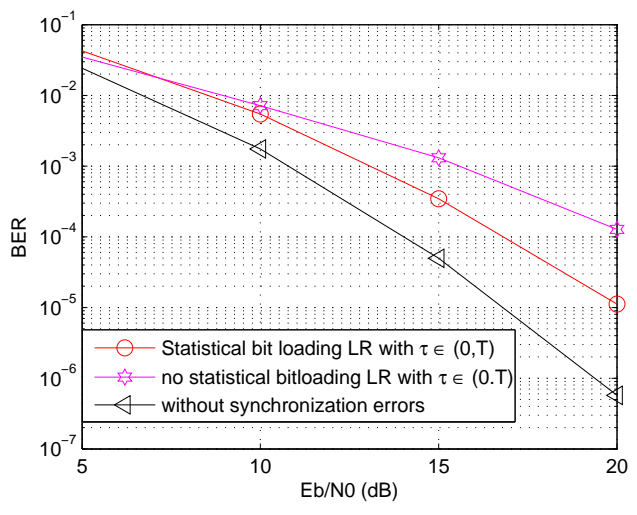

Fig. 4. Performance comparison with/without statistical bit loading

\section{CONCLUSiOnS}

A robust STBC transmission scheme to combat the timing synchronization errors over frequency-selective multiple-access channels is proposed in this paper. The synchronization errors result in an equivalent channel model with larger number of correlated channel taps. The analysis of the statistical power profile based on this correlated equivalent channel model shows that the average power on some sub-carriers are always less than the others. A robust statistical bit loading algorithm is proposed to utilize this average power profile. At the receiver, using the structure of Alamouti STBC, a closedform QR decomposition of the equivalent channel matrix is derived which simplifies computation complexity of all the interference cancellation schemes involving QR decomposition. Simulation results show that our proposed scheme achieves $3.5 \mathrm{~dB}$ SNR gain at $\mathrm{BER}=10^{-4}$.

\section{REFERENCES}

[1] R.L.Choi, M. T. Ivrlac, R. D. Murch, and W. Utschick, On strategies of multiuser MIMO transmit signal processing, IEEE Trans. on Wireless Communications, vol. 3, no. 6, pp. 1936 1941, Nov. 2004.

[2] N. Al-Dhahir, Overview and comparison of equalization schemes for space-time-coded signals with application to edge, in IEEE Transactions on Signal Processing Special Issue, October 2002.

[3] W. Zhang and X. Ma, Performance analysis for V-BLAST systems with linear equlization, CISS, JHU, March, 2005

[4] R. F. H. Fisher and J. B. Huber, A new loading algorithm for discrete multitone transmission, Global Telecommunications conference, 1996, Globecom96.

[5] Y. Mei,Y. Hua, A. Swami and B. Daneshrad, Combating synchronization errors in cooperative relays, IEEE International Confereence on Acoustics, Speech, and Signal Processing, Vol 3,, March 2005 18-23.

[6] A.F. Naguib, N. Seshadri, and A.R. Calderbank, Applications of space-time block codes and interference suppression for high capacity and high data rate wireless systems, in Proc. 32nd Asilomar Conf. Signals, Systems, and Computers Pacific Grove, CA, Vol. 2, Nov. 1998, pp: 1803-1810.

[7] S.M. Alamouti, A simple transmit diversity technique for wireless communications, IEEE Journal on Selected Areas in Communications, Vol 16, Issue 8, Oct. 1998, pp:1451 - 1458.

[8] J. G. Proakis, Digital Communications, McGraw-Hill, 4th edition, 2001.

[9] A.H. Sayed, W.M. Younis, and A. Tarighat, An invariant matrix structure in multiantenna communications, IEEE Signal Processing Letters, Volume 12, Issue 11, Nov. 2005, pp:749 - 752.

[10] A. Stamoulis, N. Al-Dhahir, and A.R. Calderbank, Further Results on Interference Cancellation for Space-Time Block-Coded Systems, Asilomar, November 2001 